\title{
APLICATIVOS BANCÁRIOS: PROPOSTA DE UM MODELO ESTRUTURAL DE PERCEPÇÃO DE ATRIBUTOS
}

\author{
BANKING APPLICATIONS: PROPOSAL OF A STRUCTURAL MODEL OF \\ ATTRIBUTES PERCEPTIONS
}

Antônio Soares de Siqueira Neto

Centro Universitário UNA - Brasil

ORCID ID: http://orcid.org/0000-0002-1634-5834

antonysoa@gmail.com

Danilo de Melo Costa

Centro Universitário UNA - Brasil

ORCID ID: http://orcid.org/0000-0002-3001-0352 danilomct@gmail.com
Submissão: 21//1/20/9. Aceitação: 01/02/2020.

Publicação: 31/03/2020. Sistema de avaliação: Double

blind review. Universidade FUMEC / FACE, Belo Horizon-

te - MG, Brasil. Editores Prof. Dr. Mário Teixeira Reis Neto

- Prof. Dr Cid Gonçalves Filho.

\section{RESUMO}

O mercado bancário está em constante evolução, Recentemente, notou-se um expressivo crescimento dos aplicativos bancários utilizados nos smartphones. Este crescimento faz com que as instituições financeiras busquem compreender as necessidades aprimoramento por meio da experiência dos clientes.A partir do exposto, objetivou-se compreender as principais características destes apps como forma de sugerir um modelo estrutural integrador. Foi realizada uma pesquisa quantitativa com 399 respondentes no estado de Minas Gerais, considerando as cinco maiores instituições bancárias do Brasil. Os dados receberam tratamento estatístico por meio de análise fatorial, definindo-se construtos de terceira ordem (segurança, confiabilidade, eficiência, comunicação, facilidade e comodidade), segunda ordem (percepções sistêmicas e percepções de interação) e primeira ordem (percepções gerais de qualidade). Os resultados obtidos demonstram que, de uma forma geral, os clientes tendem a concordar sobre a importância de todos estes constructos, com maior média de concordância nos itens de comodidade e segurança e uma menor média nos itens de eficiência e comunicação. Logo, visando à inovação neste mercado, as instituições devem buscar uma proposta integradora destes itens, como forma de obter vantagem competitiva e evolução neste mercado.

\section{PALAVRAS-CHAVE}

Aplicativos bancários. Inovação. Competitividade. Percepções sistêmicas. Percepções de interação. 


\section{ABSTRACT}

The banking market is constantly evolving. Recently, there has been a significant growth in banking applications used on smartphones. This growth makes financial institutions seek to understand the needs for improvement through the experience of customers. Based on the above, the objective was to understand the main characteristics of these apps as a way to suggest an integrating structural model. A quantitative survey was carried out with 399 respondents in the state of Minas Gerais, considering the five largest banking institutions in Brazil. The data received statistical treatment through factor analysis, defining constructs of third order (security, reliability, efficiency, communication, ease and convenience), second order (systemic perceptions and perceptions of interaction) and first order (general perceptions of quality). The results obtained demonstrate that, in general, customers tend to agree on the importance of all these constructs, with a higher average of agreement on the items of convenience and safety and a lower average on the items of efficiency and communication. Therefore, aiming at innovation in this market, institutions should seek a proposal that integrates these items, as a way to obtain competitive advantage and evolution in this market.

\section{KEYWORDS}

Banking applications. Innovation. Competitiveness. Systemic perceptions. Perceptions of interaction.

\section{INTRODUÇÃO}

A evolução nas tecnologias bancárias meIhorou as demandas de processos e o dia a dia dos clientes, reduzindo a necessidade de ir a uma agência para resolução de seus problemas, conseguindo resolver parte das demandas através de aplicativos em seus celulares. Este processo de mudança na comunicação se mostrou possível em decorrência da expansão das TIC's, após os anos 1990, com um grande número de pessoas conectadas à internet e com a possibilidade de se criar formas alternativas de atendimento aos clientes (MAINETTI JUNIOR; GRAMANI; BARROS, 20I4). Neste viés de evolução, os app's se tornaram parte integrante do setor bancário, gerando impactos não somente em relação aos bancos e seus clientes, mas também gerando um case de sucesso para os demais mercados.

Observa-se dentro desta perspectiva que a utilização dos aplicativos nos celulares, de acordo com a Febraban (2017), está em constante crescimento e ressaltase que em 2013 a utilização deste tipo de serviço representava $4 \%$ no que se refere às transações bancárias. Em recente atualização de seus relatórios, a Febraban (2018) informa o crescimento para $10 \%$ em 2014 , $20 \%$ em 20I5, 27\% em 2016 e em 2017 com índices no patamar de 35\%. Nesta análise, constata-se que as novas tecnologias apresentadas pelas instituições bancárias vêm mudando os hábitos dos consumidores. Com estes números, ressalta ainda o relatório que atualmente os mobile's são a 
principal forma de intermediação entre os bancos e seus clientes, com 25,6 bilhões de transações no ano de 2017, apresentando crescimento em vários serviços e produtos, como contratação de crédito (crescimento de $141 \%$ ), investimento (crescimento de $53 \%$ ), solicitação de cartões de crédito (crescimento de 53\%) emissão de DOC/TED (crescimento de $45 \%$ ) e pagamento de contas (crescimento de 85\%), todos em comparação ao ano anterior. Esta mudança na forma de transacionar vem sendo impactada pelos grandes investimentos feitos na área de tecnologia, chegando a 19,5 bilhões em 2017 (aumento de 5\% em comparação ao ano anterior), gerando uma maior confiabilidade para seus usuários (FEBRABAN, 20I8).

Assim, percebe-se que ano após ano, a utilização dos aplicativos bancários vem crescendo e tornando-se a opção preferida entre os usuários dos bancos. Desta forma, mostra-se necessário um estudo sobre seus principais atributos e a criação de um modelo estrutural para melhor entender seus reflexos de inovação do setor financeiro, assim como analisar as percepções dos clientes, geradoras de reflexos em sua utilização dos app's.

\section{REFERENCIALTEÓRICO}

Analisando a forma que as novas tecnologias surgem e se tornam obsoletas rapidamente, percebe-se a necessidade das instituições financeiras em buscar novas parcerias para que consigam criar constantemente mecanismos de comunicação e interação eficazes no contato com seus clientes.

Neste contexto, a cada nova tecnologia que surge, se destrói conceitos anteriores e cria-se a necessidade das empresas em modificar suas demandas, onde este pro- cesso de destruição criativa gera a possibilidade de um constante movimento de inovação para o mercado, com a possibilidade de se criar ou destruir organizações, mudando sua estrutura (LOPES, 2016; SCHUMPETER, 1942).

Ressalta-se ainda que as empresas estão evoluindo a todo momento, onde é necessário observar, dentre outras questões, a estrutura do mercado, com suas novas oportunidades e tendências. Neste quesito, a diferenciação de produtos (que trata-se da apresentação de produtos ou serviços que substituam outros produtos, com apresentação de um melhor resultado) e diversificação (que se trata de inclusão de uma nova gama de produtos ou serviços que antes não eram ofertados), fazem parte de uma estratégia competitiva, que pode manter uma organização em condições de se destacar (LOPES, 2016; SCHERER, 1996).

Assim, a criação de conexões e parcerias para que se possa instituir as diferenciações necessárias para se manterem atuais no segmento bancário se faz presente. Desta forma, se mostra assertivo o caminho de se aproximar das organizações distintas de seu foco de atuação, para possibilitar a criação destes processos de inovação a partir de conceitos não vislumbrados internamente, criando novos laços conforme veremos a seguir (GRANOVETTER, 1973; KAUFMAN, 20I2).

\section{Diferenciais competitivos a partir de novas parcerias e novos relacionamentos}

Conforme explica Power (20I4), as organizações que não conseguem criar novos laços em diversas áreas poderão rapidamente ser absorvidas pelo mercado. Neste sentido, entende-se que as companhias devem cada 
vez mais sair de sua área de conforto, para buscar novas parcerias e, com isso, propiciar novas experiências para seus clientes.

Com este entendimento, tem-se que as organizações precisam focar em seus "laços fracos" para que possam adquirir conhecimentos e, com isso, poder gerar novos diferenciais competitivos, criando inovações ao seu mercado de atuação, com novas perspectivas ou novas concepções, que em um primeiro momento podem não parecer ligadas ao negócio da organização, mas que ao se analisar mais profundamente pode gerar um diferencial competitivo não pensado pelo mercado (GRANOVETTER, 1973; KAUFMAN, 2012 ).

Assim, entende-se que a empresa buscando contato com seus "laços fracos”, poderá localizar a existência e criar pontes entre os possíveis "buracos estruturais" existentes entre elas, gerando a possibilidade de se contribuir para seus processos de inovação. Com isto, a aproximação entre empresas de diversas áreas de conhecimento se mostra necessária, para que seja possível, em contato com equipes que idealizam processos diferentes, se criar novas ideias para sua organização (BURT, I993; CARVALHO; SERIO; VASCONCELLOS, 2012).

Neste sentido, pode-se ressaltar que uma das parcerias exitosas foram entre os bancos com setores de TIC's (tecnologias da informação e comunicações), em que a partir do advento da internet, as organizações tiveram que pensar em seu negócio fora de seu espaço físico, conectando-se com empresas diversas, para trazer soluções antes não pensadas. Esta união permitiu que as organizações explorassem estas lacunas, o que ocasionou em várias inovações para o setor, tendo entre elas os aplicativos bancários, que vem crescendo no mercado financeiro.
Desta forma, nota-se que as despesas com tecnologia se mostram substanciais e são um investimento necessário para a manutenção das organizações no mercado bancário, interferindo na eficiência destas instituições, principalmente no setor varejista, impactando diretamente a organização e podendo gerar um diferencial competitivo (MAINETTI JUNIOR; GRAMANI; BARROS, 20I4).

Ainda analisa Bombonatti Filho et. al. (20l3) que o setor bancário cresceu muito nos primeiros onze anos do século $\mathrm{XXI}$, onde o número de clientes evoluiu em decorrência das facilidades de acesso, tanto quanto pelas novas formas de relacionamento que estas instituições apresentaram.

Logo, observa-se que a busca para criar novos laços, fora dos padrões, muitas vezes considerados convencionais, mostra-se necessário para que as instituições apresentem um diferencial e possam criar novas vantagens competitivas.

\section{A integração com novos mercados para a criação de diferenciais competitivos}

Com base nos processos evolutivos no setor bancário, as empresas evoluíram muito no último século, mudando completamente a forma de se comunicar e estruturar o relacionamento com seus clientes. Ocorre que a cada dia se torna mais difícil criar vantagens competitivas neste mercado, sendo que a busca de novas parcerias, novas ferramentas ou novas formas de se comunicar se mostra uma necessidade para o crescimento deste setor.

Nestas condições, Kappel, Arruda e Pimenta (20l4) analisam o comportamento dos clientes que não utilizam os serviços pelos canais remotos, o que gera um au- 
mento de custo para as organizações, em que os clientes apontam motivos para a não utilização destes serviços, que passam pela confiança na ferramenta até a dificuldade de se atender por estes canais.

As grandes organizações bancárias podem, dentro desta análise, perceber uma oportunidade para este grupo de clientes ainda não digitais, que podem reduzir seus custos e aumentar sua produtividade, onde há a possibilidade de se criar mecanismos de autoatendimento menos complexos e mais simples para captar um perfil de cliente ainda não adepto a esta nova forma de relacionamento.

Logo, as empresas devem buscar uma nova curva de crescimento pela inovação, criando vantagens competitivas a todo momento, visto que toda vantagem criada é temporária e as organizações que visam se manter com diferenciais em relação aos seus concorrentes devem se reinventar sempre, evitando que seus processos se tornem obsoletos (POWER, 20I4).

\section{Evolução das TIC's - Mudanças na forma de atendimento para um modelo digital e seus desafios}

No atual momento das organizações a utilização de ferramentas tecnológicas é necessária para a manutenção das empresas no mercado de forma competitiva. Analisando desta forma, Malaquias e Albertin (20II) explicam que as organizações, atualmente, necessitam investir continuadamente em novas fontes de conhecimento tecnológico, agregando valor aos processos existentes, criando um imaginário de redução de custos para o cliente ou aumento da qualidade no produto ofertado.

Nesta ótica, no contexto da evolução das TIC's (tecnologias da informação e co- municações), as organizações passaram no decorrer dos anos a depender de forma excessiva destes processos e sua contribuição passou a ser essencial para entender melhor os processos de inovação que estas tecnologias trazem para as empresas, onde os diferenciais competitivos criados pelas organizações dificilmente surgem fora das inovações tecnológicas (ALBERTIN; ALBERTIN, 20 I2; TESTA; LUCIANO; JAEGER NETO, 20I3).

Neste contexto, Bombonatti Filho et. al., (20I3) verificam que as empresas bancárias acompanharam estes processos de inovação criados pelo mercado, com foco na redução de custos operacionais e facilitação nos processos de atendimento, o que se mostrou no decorrer dos anos como uma estratégia assertiva destas organizações, concentrando cerca de $5 \%$ do total do volume investido anualmente no mundo em tecnologia.

Desta forma, os investimentos criados pelas TIC's foram essenciais para a colocação das empresas financeiras no patamar de eficiência que se encontram hoje e balizaram as tecnologias e forma de atendimento remota que apresentam atualmente, criando condições de a cada dia realizar melhorias nos processos de interação com os seus clientes.

\section{Variáveis para aplicativos bancários: modelo teórico}

No mercado financeiro, a utilização de aplicativos bancários já se mostra uma realidade que visa a diminuição de custos operacionais e aumento de produtividade das organizações, conforme já discutido nos tópicos anteriores. Nesta vertente, se verificou quais variáveis são importantes para o desenvolvimento desta tecnologia para 
estas empresas e, a partir disso, ser a base para a pesquisa quantitativa que foi realizada posteriormente.

A usabilidade de um celular apresenta importância no processo de utilização de seus softwares e esse processo evolui constantemente. Com isto é necessário a evolução dos designs e projetos apresentados, com foco na concepção e avaliação dos produtos (HEO et al., 2009; OLSINA; SANTOS; LEW, 20I4).

Nesta realidade, a usabilidade também se mostra um fator a ser analisado na perspectiva dos aplicativos bancários. Conforme explicam Kappel, Arruda e Pimenta (2014) vários usuários ainda apresentam dificuldades na utilização aos meios remotos.

Assim, percebe-se que a usabilidade de um aplicativo é analisada sob vários aspectos, tendo destaque a velocidade, interatividade, tolerância a erros, menor consumo de armazenamento, dependência de plataforma, eficácia, desempenho, consumo de bateria, estética, eficiência e facilidade de uso (GOEL; NAGPAL; MEHROTRA, 2018). Esta usabilidade cria condições de verificação para a análise da eficiência, segurança, comodidade, facilidade e confiabilidade das operações.

Este fator gera preocupação nos clientes quanto à segurança das transações, bem como os constantes riscos operacionais que geram receios em lançar informações nestes app's. Assim, em alguns casos a confiabilidade das operações também é colocada em dúvida, o que precisa ser analisada pelas instituições financeiras, sendo, também um critério de análise desta pesquisa (KAPPEL;ARRUDA; PIMENTA, 20 I4).

A eficiência é outro fator de importância para os clientes, conforme explicam Kaplan e Haenlein (20l0), visto que a atual forma de comunicação rápida e eficaz da internet exige que os aplicativos das grandes organizações também sejam da mesma forma. Assim a eficiência dos app's quanto a sua agilidade e apresentação de soluções diretamente dos smartphones se tornam necessárias no atual momento da sociedade e pelo desejo de inovação de seus clientes.

Desta forma, observa-se que a segurança, confiabilidade nos sistemas de interação e eficiência são itens importantes para os aplicativos bancários, que podem ser analisados sob a ótica de percepções sistêmicas.

Sob outro foco, percebe-se ainda, conforme explica Kaplan e Haenlein (2010), que a comunicação evoluiu bastante nas últimas décadas, se tornando mais rápida e eficaz, sendo um ponto importante para as organizações bancárias, assim como para seus aplicativos de comunicação. Desta forma, um sistema de interação que seja eficiente para interligar as ideias dos clientes e da empresa se faz necessário, sendo, portanto, um ponto importante para a evolução e utilização dos sistemas de relacionamento remoto.

Nota-se também que a facilidade em efetuar as transações também se mostra como variável importante para os clientes bancários, sendo construto a ser considerada na decisão de utilizar ou não os aplicativos bancários (FIEBIG; FREITAS, 20I I ; KAPPEL;ARRUDA; PIMENTA, 20।4; SIQUEIRA NETO; BARCELOS; COSTA, 2018).

Ressalta-se, por fim, que a comodidade oferecida pelos aplicativos bancários também é um fator importante para a utilização dos clientes aos app's das instituições financeiras, com o foco no autoatendimento e independência na realização das tarefas necessárias, não sendo imperioso ir até uma agência física para a realização de transações financeiras. Fiebig e Freitas 
(20II) ressaltam ainda que vários clientes optam pelo contato pessoal na rede de agências, preferindo este à comodidade oferecida pelos aplicativos, que mostra que esta variável é importante para o entendimento dos motivos que os clientes ainda preferem ou não utilizar estes dispositivos.

Assim, a comunicação, a facilidade e a comodidade na utilização dos aplicativos bancários são itens de importância na interligação dos consumidores às instituições financeiras, sendo analisados sob a ótica da percepção de interação dos clientes.

Assim, buscando entender de forma mais ampla o sentimento dos usuários quanto aos aplicativos bancários, foram utilizadas as variáveis localizadas no decorrer do trabalho, com foco nas percepções sistêmicas, voltadas para a avaliação da segurança, confiabilidade e eficiência das operações, assim como a análise das percepções de intera- ção, com análise na comunicação, facilidade e comodidade das operações, conforme modelo apresentado a seguir:

Logo, foram observados através de pesquisa quantitativa as variáveis que interferem na qualidade dos aplicativos bancários, buscando entender quais delas possuem maior peso na avaliação dos clientes, assim como quais são essenciais para a evolução destes dispositivos.

\section{METODOLOGIA}

Para este estudo, foi utilizada a técnica de estudo de caso que, conforme explica Yin (2015), esta estratégia visa entender entre outras questões, os fenômenos organizacionais e sociais, buscando compreender determinadas questões complexas e que precisem de um aprofundamento para sua melhor interpretação.

Em análise a esta pesquisa, o estudo de

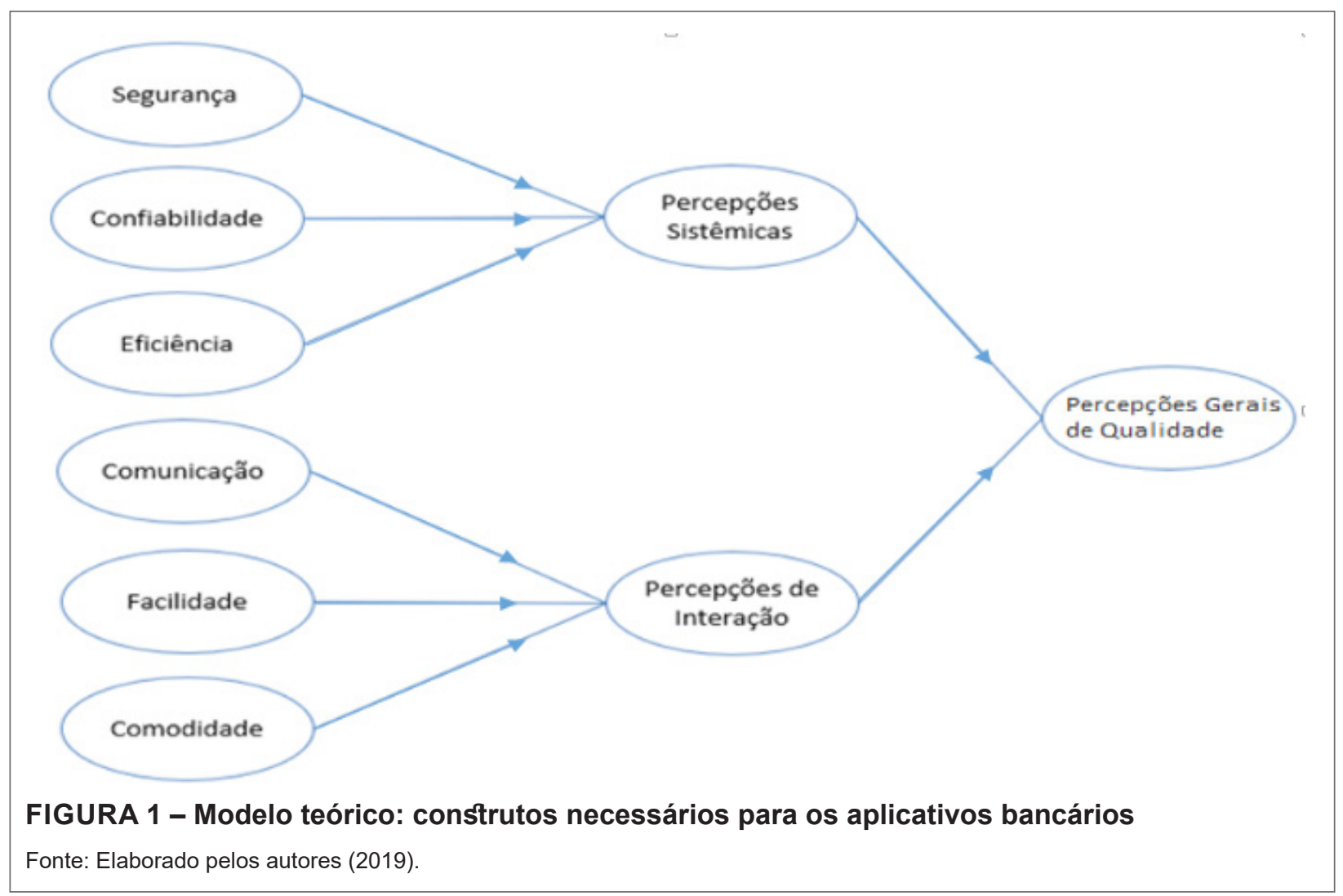


caso é ideal para o entendimento de um setor específico que foi estudado, qual sendo, o setor bancário e suas instituições, assim como o comportamento de seus clientes.

Para a execução da análise de dados, a pesquisa foi realizada em duas fases. $\mathrm{Na}$ primeira fase compreenderam-se as principais mudanças do setor estudado sendo utilizada a pesquisa documental e bibliográfica e a partir disso estruturar um modelo teórico conceitual com as principais variáveis a serem consideradas no desenvolvimento de aplicativos bancários. $\mathrm{Na}$ segunda fase realizou-se uma pesquisa junto aos clientes bancários, buscando entender as perspectivas e percepções destes consumidores quanto aos serviços bancários e seus reflexos futuros, utilizando-se do modelo de questionário, que é aquele que prevê uma experiência de conhecimento com roteiro estabelecido previamente, visando uma padronização das perguntas realizadas, com o intuito de se comparar suas respostas, verificando os padrões de suas percepções, assim como testar hipóteses e explicar objetivos específicos, conseguindo descrever situações de forma quantitativa (GIL, 2008; MARCONI; LAKATOS, 20 I7)

Tendo por base a população analisada, qual seja, clientes que possuem contas bancárias, foi baseada essa pesquisa nas cinco maiores instituições nacionais, pois estas concentram a maior parte dos clientes com ativos, com representatividade em torno de $93 \%$ do mercado, sendo que estas empresas possuem um total de 358.979.447 de clientes (BACEN, 20I8).

Para análise de dados, foi realizada uma pesquisa descritiva e quantitativa com os clientes das instituições financeiras. Para esta pesquisa, foi aplicado um questionário estruturado, com grau de confiança de
95\%, e margem de erro de 5\%. De acordo com os cálculos propostos por Stevenson (200 I), para uma população de 358.979.447 clientes, o total da amostra deveria ser superior a 385 respondentes.

A pesquisa foi realizada com 550 (quinhentos e cinquenta) indivíduos, entre o período de 12 de julho de 2018 a 17 de agosto de 2018, ressaltando-se que para esta pesquisa foram eliminados o respondentes que não utilizam aplicativos bancários, sendo o total de 88 (oitenta e oito) respondentes, assim como os respondentes de outros estados (pesquisa focada no estado de Minas Gerais), totalizando 63 (sessenta e três) questionários, tendo um total de 399 questionários válidos para análise. $O$ instrumento de coleta de dados foi um questionário estruturado com escala tipo Likert variando de I (Discordo Totalmente) a 7 (Concordo Totalmente). Dessa forma, intervalos estritamente menores que 4 indicam que os indivíduos tendem a discordar, enquanto intervalos estritamente maiores que 4 indicam que os indivíduos tendem a concordar e que intervalos que contém 4 indicam que não tenderam a discordar nem concordar.

Foi utilizado como forma de interpretação de dados, a análise estatística que, conforme explica Gerhardt e Silveira (2009), consiste no processamento de informações através de cálculos matemáticos, organizados por gráficos e/ou tabelas, sendo esta análise quantitativa, utilizada a análise fatorial, criados itens de primeira ordem (segurança, confiabilidade, eficiência, comunicação, facilidade e comodidade), segunda ordem (percepções sistêmicas e percepções de interação) e terceira ordem (percepções gerais de qualidade).

Logo, foi criado um modelo teórico con- 
ceitual gerado após a fase I, recebendo as respectivas cargas a partir da aplicação dos questionários, de forma a apresentar para as instituições financeiras quais aspectos são considerados mais relevantes pelo seu público alvo no que se refere aos aplicativos bancários, representando oportunidades de inovação.

\section{RESULTADOS}

A seguir, são apresentados os resultados obtidos com a pesquisa realizada.

\section{Análise Fatorial}

A análise fatorial foi utilizada para criação dos indicadores de primeira, segunda e terceira ordem. Cabe ressaltar que foram analisadas a validade convergente, a confiabilidade, a adequação da análise fatorial e a dimensionalidade dos indicadores. Foram analisados de forma fatorial os constructos de primeira, segunda e terceira ordem, conforme abaixo descritas.

\section{Indicadores de primeira ordem}

$\mathrm{Na}$ Tabela I podem ser verificadas as cargas fatoriais, as comunalidades e os pesos da análise fatorial.

O item CF6 ("Considero que novas formas de segurança, como reconhecimento facial (pela digital ou íris) são importantes para a confiabilidade das operações realizadas pelos aplicativos bancários.") foi retirado do constructo Confiabilidade por apresentar carga fatorial menor que 0,50 , enquanto o item CO4 ("Penso que a comunicação através dos aplicativos bancários é mais eficiente do que a realizada através das agências físicas.") foi retirado do constructo Comunicação, pois estava impedindo a validação do mesmo. Observa-se que no modelo final todas as cargas foram satisfatórias, ou seja, maiores que 0,50 .
Os resultados para a validade e qualidade dos constructos de primeira ordem são apresentados a seguir.

Observou-se que todos os constructos apresentaram valores de Alfa de Cronbach (A.C.) ou Confiabilidade Composta (C.C.) acima de 0,60 , evidenciando assim a confiabilidade dos mesmos. Os valores de KMO foram maiores que 0,50 em todos os constructos, o que indica que o ajuste da Análise Fatorial foi adequado. De acordo com o critério Acceleration Factor, todos os constructos foram unidimensionais. Houve validação convergente em todos os constructos $(A \bigvee E s>0,40)$. Houve validação discriminante para todos os constructos, uma vez que as variâncias compartilhadas máximas foram menores que as respectivas AVEs.

\section{Descrição dos indicadores de primeira ordem}

Os resultados abaixo apresentam a descrição dos indicadores de primeira ordem criados a partir da Análise Fatorial.

a) Percepções Sistêmicas

Ao se verificar o constructo de percepções sistêmicas, após as devidas análises fatoriais, apresentou-se os seguintes resultados:

Em verificação às percepções sistêmicas, nota-se que o item segurança apresentou a maior média, com indicador de 6,29 acompanhado pela confiabilidade, com índice de 6,15 e ambos dentro do limite do intervalo de confiança, o que demonstra que os clientes tendem a valorizar mais um aplicativo que garanta aos seus consumidores que as operações realizadas são, de alguma forma, acompanhadas e certificadas, evitando fraudes bancárias, gerando uma sensação de fidúcia entre as partes.

O constructo eficiência apresentou a menor média, com índice de 5,76, onde os 
TABELA 1 - Análise Fatorial dos constructos de primeira ordem em pesquisa realizada junto aos clientes bancários em 2018

\begin{tabular}{|c|c|c|c|c|c|c|c|c|}
\hline \multirow{2}{*}{\multicolumn{2}{|c|}{ Constructo }} & \multirow{3}{*}{$\begin{array}{l}\text { Itens } \\
\text { SE1 }\end{array}$} & \multicolumn{3}{|c|}{ Modelo Inicial } & \multicolumn{3}{|c|}{ Modelo Final } \\
\hline & & & \multirow{2}{*}{$\begin{array}{l}\text { C.F. }{ }^{1} \\
0,63\end{array}$} & \multirow{2}{*}{$\begin{array}{c}\text { Com. }^{2} \\
0,39\end{array}$} & \multirow{2}{*}{$\begin{array}{c}\text { Peso } \\
0,20\end{array}$} & \multirow{2}{*}{$\begin{array}{l}\text { C.F. }{ }^{1} \\
0,63\end{array}$} & \multirow{2}{*}{$\begin{array}{c}\text { Com. }^{2} \\
0,39\end{array}$} & \multirow{2}{*}{$\begin{array}{r}\text { Peso } \\
0,20\end{array}$} \\
\hline \multirow{21}{*}{$\begin{array}{l}\text { Percepções } \\
\text { Sistêmicas }\end{array}$} & \multirow{7}{*}{ Segurança } & & & & & & & \\
\hline & & SE2 & 0,61 & 0,37 & 0,20 & 0,61 & 0,37 & 0,20 \\
\hline & & SE3 & 0,79 & 0,63 & 0,25 & 0,79 & 0,63 & 0,25 \\
\hline & & SE4 & 0,65 & 0,42 & 0,21 & 0,65 & 0,42 & 0,21 \\
\hline & & SE5 & 0,70 & 0,49 & 0,23 & 0,70 & 0,49 & 0,23 \\
\hline & & SE6 & 0,63 & 0,40 & 0,20 & 0,63 & 0,40 & 0,20 \\
\hline & & SE7 & 0,64 & 0,40 & 0,20 & 0,64 & 0,40 & 0,20 \\
\hline & \multirow{7}{*}{ Confiabilidade } & CF1 & 0,67 & 0,45 & 0,25 & 0,64 & 0,41 & 0,25 \\
\hline & & CF2 & 0,60 & 0,36 & 0,23 & 0,64 & 0,41 & 0,25 \\
\hline & & CF3 & 0,67 & 0,45 & 0,25 & 0,71 & 0,50 & 0,28 \\
\hline & & CF4 & 0,62 & 0,38 & 0,23 & 0,63 & 0,39 & 0,24 \\
\hline & & CF5 & 0,70 & 0,50 & 0,27 & 0,70 & 0,48 & 0,27 \\
\hline & & CF6 & 0,37 & 0,14 & 0,14 & - & - & - \\
\hline & & CF7 & 0,62 & 0,38 & 0,23 & 0,61 & 0,37 & 0,24 \\
\hline & \multirow{7}{*}{ Eficiência } & EF1 & 0,65 & 0,43 & 0,23 & 0,65 & 0,43 & 0,23 \\
\hline & & EF2 & 0,68 & 0,46 & 0,24 & 0,68 & 0,46 & 0,24 \\
\hline & & EF3 & 0,70 & 0,50 & 0,25 & 0,70 & 0,50 & 0,25 \\
\hline & & EF4 & 0,66 & 0,44 & 0,23 & 0,66 & 0,44 & 0,23 \\
\hline & & EF5 & 0,59 & 0,35 & 0,21 & 0,59 & 0,35 & 0,21 \\
\hline & & EF6 & 0,62 & 0,38 & 0,21 & 0,62 & 0,38 & 0,21 \\
\hline & & EF7 & 0,57 & 0,32 & 0,20 & 0,57 & 0,32 & 0,20 \\
\hline \multirow{21}{*}{$\begin{array}{l}\text { Percepções } \\
\text { de Interação }\end{array}$} & \multirow{7}{*}{ Comunicação } & CO1 & 0,60 & 0,36 & 0,22 & 0,64 & 0,41 & 0,25 \\
\hline & & $\mathrm{CO} 2$ & 0,66 & 0,43 & 0,24 & 0,70 & 0,48 & 0,28 \\
\hline & & $\mathrm{CO} 3$ & 0,60 & 0,35 & 0,22 & 0,59 & 0,35 & 0,24 \\
\hline & & $\mathrm{CO} 4$ & 0,53 & 0,28 & 0,20 & - & - & - \\
\hline & & CO5 & 0,67 & 0,45 & 0,25 & 0,63 & 0,39 & 0,25 \\
\hline & & $\mathrm{CO} 6$ & 0,54 & 0,29 & 0,20 & 0,56 & 0,31 & 0,22 \\
\hline & & $\mathrm{CO} 7$ & 0,73 & 0,54 & 0,27 & 0,75 & 0,56 & 0,30 \\
\hline & \multirow{7}{*}{ Facilidade } & FA1 & 0,65 & 0,42 & 0,20 & 0,65 & 0,42 & 0,20 \\
\hline & & FA2 & 0,69 & 0,47 & 0,21 & 0,69 & 0,47 & 0,21 \\
\hline & & FA3 & 0,64 & 0,40 & 0,20 & 0,64 & 0,40 & 0,20 \\
\hline & & FA4 & 0,69 & 0,47 & 0,21 & 0,69 & 0,47 & 0,21 \\
\hline & & FA5 & 0,62 & 0,39 & 0,19 & 0,62 & 0,39 & 0,19 \\
\hline & & FA6 & 0,74 & 0,54 & 0,23 & 0,74 & 0,54 & 0,23 \\
\hline & & FA7 & 0,71 & 0,50 & 0,22 & 0,71 & 0,50 & 0,22 \\
\hline & \multirow{7}{*}{ Comodidade } & CD1 & 0,85 & 0,71 & 0,19 & 0,85 & 0,71 & 0,19 \\
\hline & & CD2 & 0,76 & 0,57 & 0,17 & 0,76 & 0,57 & 0,17 \\
\hline & & CD3 & 0,85 & 0,72 & 0,19 & 0,85 & 0,72 & 0,19 \\
\hline & & CD4 & 0,89 & 0,79 & 0,20 & 0,89 & 0,79 & 0,20 \\
\hline & & CD5 & 0,72 & 0,51 & 0,16 & 0,72 & 0,51 & 0,16 \\
\hline & & CD6 & 0,78 & 0,61 & 0,18 & 0,78 & 0,61 & 0,18 \\
\hline & & CD7 & 0,70 & 0,49 & 0,16 & 0,70 & 0,49 & 0,16 \\
\hline
\end{tabular}

Fonte: Elaborado pelos autores (2019). 
TABELA 2 - Validação dos constructos de primeira ordem em pesquisa realizada com clientes bancários em 2018

\begin{tabular}{|c|c|c|c|c|c|c|c|c|}
\hline \multicolumn{2}{|l|}{ Constructos } & Itens & A.C. ${ }^{1}$ & C.C. ${ }^{2}$ & $\mathrm{KMO}^{3}$ & $\operatorname{Dim}^{4}$ & AVE $^{5}$ & VCM $^{6}$ \\
\hline \multirow{3}{*}{ Percepções Sistêmicas } & Segurança & 7 & 0,77 & 0,79 & 0,85 & 1 & 0,44 & 0,31 \\
\hline & Confiabilidade & 6 & 0,71 & 0,76 & 0,77 & 1 & 0,43 & 0,31 \\
\hline & Eficiência & 7 & 0,75 & 0,78 & 0,70 & 1 & 0,41 & 0,27 \\
\hline \multirow{3}{*}{ Percepções de Interação } & Comunicação & 6 & 0,71 & 0,75 & 0,75 & 1 & 0,42 & 0,27 \\
\hline & Facilidade & 7 & 0,79 & 0,80 & 0,80 & 1 & 0,46 & 0,26 \\
\hline & Comodidade & 7 & 0,89 & 0,88 & 0,87 & 1 & 0,63 & 0,22 \\
\hline
\end{tabular}

${ }^{1}$ Alfa de Cronbach; ${ }^{2}$ Confiabilidade Composta; ${ }^{3}$ Critério de Kaiser-Meyer-Olkin; 4Dimensionalidade; 5Variância Extraída; 6 Variância Compartilha Máxima.

Fonte: Elaborado pelos autores (2019).

TABELA 3 - Descrição dos indicadores de primeira ordem - Percepções sistêmicas, em pesquisa realizada com clientes bancários em 2018

\begin{tabular}{l|c|c|c|c}
\hline \multicolumn{1}{l|}{ Indicadores } & Média & D.P. & I.C. - 95\% \\
\hline \multirow{3}{*}{ Percepções Sistêmicas } & Segurança & 6,29 & 0,87 & {$[6,20 ; 6,37]$} \\
\cline { 2 - 5 } & Confiabilidade & 6,15 & 0,83 & {$[6,07 ; 6,24]$} \\
\cline { 2 - 5 } & Eficiência & 5,76 & 1,04 & {$[5,66 ; 5,87]$} \\
\hline
\end{tabular}

Fonte: Elaborado pelos autores (2019).

clientes tendem a valorizar menos questões voltadas à redução de espaço no celular, utilização de dados ou tempo de resposta, em comparação às questões de segurança. Assim, pode-se concluir que os clientes concordam com a necessidade de um sistema eficiente, mas desde que esta eficiência não reduza os processos de segurança das operações.

b) Percepções de Interação

Ao se verificar o constructo de percepções de interação, após as devidas análises fatoriais, se apresentou os seguintes resultados:

A comunicação apresenta um resultado menor, onde os clientes, apesar de concordarem sobre a importância de um sistema ágil no processo de interligação entre os clientes e seus respectivos bancos através dos app's, estes consumidores tendem a valorar mais os demais itens deste constructo.

A facilidade, apesar de uma média menor, também se mostra importante, visto que os clientes entendem que a utilização dos aplicativos de forma intuitiva, simples e direta é importante no processo de escolha em utilizar os aplicativos bancários em detrimento a outras formas de atendimento. A facilidade apresentou uma concordância também alta, com média de 6,09 , seguido com certa distância do item comunicação, com média de 5,30, não se encontrando também no intervalo de confiança.

A comodidade apresentou uma média superior aos demais itens, com índice de 6,53 , não apresentando, inclusive, aproximação com os demais, mesmo se considerando o intervalo de confiança.Verificou-se que os clientes apresentam uma alta valoração à comodidade, com a tendência da busca de mecanismos digitais e de atendimento remoto, visando trazer mais conforto em suas vidas, podendo realizar suas demandas no local e no horário que escolher.

\section{Indicadores de segunda ordem}

$\mathrm{Na}$ Tabela 5 podem ser verificados as cargas fatoriais, as comunalidades e os pesos da análise fatorial dos indicadores de 
TABELA 4 - Descrição dos indicadores de primeira ordem - Percepções de interação, em pesquisa realizada com clientes bancários, em 2018

\begin{tabular}{l|l|c|c|c}
\hline \multicolumn{2}{l|}{ Indicadores } & Média & D.P. & I.C. - 95\% \\
\hline \multirow{3}{*}{ Percepções de Interação } & Comunicação & 5,30 & 1,12 & {$[5,20 ; 5,41]$} \\
\cline { 2 - 5 } & Facilidade & 6,09 & 0,88 & {$[6,00 ; 6,17]$} \\
\cline { 2 - 5 } & Comodidade & 6,53 & 0,91 & {$[6,44 ; 6,61]$} \\
\hline \hline
\end{tabular}

Fonte: Elaborado pelos autores (2019).

TABELA 5 - Análise Fatorial dos constructos de segunda ordem em pesquisa realizada com clientes bancários em 2018

\begin{tabular}{|c|c|c|c|c|}
\hline Indicadores de $2^{\mathrm{a}}$ ordem & Indicadores de $1^{a}$ ordem & C.F. ${ }^{1}$ & Com. $^{2}$ & Peso \\
\hline \multirow{3}{*}{ Percepções Sistêmicas } & Segurança & 0,83 & 0,69 & 0,42 \\
\hline & Confiabilidade & 0,83 & 0,69 & 0,42 \\
\hline & Eficiência & 0,77 & 0,59 & 0,39 \\
\hline \multirow{3}{*}{ Percepções de Interação } & Comunicação & 0,75 & 0,56 & 0,42 \\
\hline & Facilidade & 0,87 & 0,75 & 0,49 \\
\hline & Comodidade & 0,69 & 0,47 & 0,38 \\
\hline
\end{tabular}

${ }^{1}$ Carga Fatorial; ${ }^{2}$ Comunalidade.

Fonte: Elaborado pelos autores (2019).

TABELA 6 - Validação dos constructos de segunda ordem em pesquisa realizada com clientes bancários em 2018

\begin{tabular}{l|c|c|c|c|c|c|c}
\hline Indicadores de 2a ordem & Itens & A.C. & C.C. $^{\mathbf{2}}$ & KMO $^{3}$ & Dim. $^{4}$ & AVE $^{\mathbf{3}}$ & VCM $^{6}$ \\
\hline Percepções Sistêmicas & 3 & 0,73 & 0,77 & 0,67 & 1 & 0,65 & 0,44 \\
\hline Percepções de Interação & 3 & 0,64 & 0,74 & 0,56 & 1 & 0,59 & 0,44 \\
\hline
\end{tabular}

${ }^{1}$ Alfa de Cronbach; ${ }^{2}$ Confiabilidade Composta; ${ }^{3}$ Critério de Kaiser-Meyer-Olkin; 4Dimensionalidade; 5Variância Extraída; 6 Variância Compartilha Máxima.

Fonte: Elaborado pelos autores (2019).

segunda ordem. A partir dela pode-se verificar que todas as cargas fatoriais foram maiores que 0,50 .

Os resultados para a validade e qualidade dos constructos de segunda ordem são apresentados na Tabela 6.

Ambos os constructos apresentaram valores de Alfa de Cronbach (A.C.) ou Confiabilidade Composta (C.C.) acima de 0,60 , evidenciando assim a confiabilidade dos mesmos. Os valores de KMO foram iguais a $0,50 \mathrm{em}$ ambos os constructos, $O$ que indica que ajuste da Análise Fatorial foi adequado. De acordo com o critério Acceleration Factor, ambos os constructos foram unidimensionais. Houve validação convergente em ambos os constructos, visto que as AVEs foram superiores a 0,40. De acordo com o critério de Fornell e Larcker (198I), houve validação discriminante para ambos os constructos, uma vez que as variâncias compartilhadas máximas foram menores que as respectivas AVEs.

\section{Descrição dos indicadores de segunda ordem}

Ao verificar os constructos de segunda ordem, observa-se a seguinte análise fatorial e seus respectivos desvios padrões.

Dessa forma, pode-se verificar que o indicador Percepções Sistêmicas apresentou uma média maior que a do indicador Percepções de Interação. Ressalta-se que pelo intervalo de confiança apresentado, ambos podem ser considerados estatisticamente equivalentes, no limite do desvio padrão. 
TABELA 7 - Descrição dos indicadores de segunda ordem em pesquisa realizada com clientes bancários em 2018

\begin{tabular}{l|c|c|c}
\hline Indicadores & Média & D.P. & I.C. - 95\% \\
\hline Percepções Sistêmicas & 6,08 & 0,74 & {$[6,01 ; 6,15]$} \\
\hline Percepções de Interação & 5,96 & 0,75 & {$[5,88 ; 6,03]$} \\
\hline
\end{tabular}

Fonte: Elaborado pelos autores (2019).

\section{Indicadores de terceira ordem}

$\mathrm{Na}$ Tabela 8 podem ser verificadas as cargas fatoriais, as comunalidades e os pesos da análise fatorial do indicador de terceira ordem.A partir dela pode-se verificar que todas as cargas fatoriais foram maiores que 0,50 .

Os resultados para a validade e qualidade do constructo de terceira ordem são apresentados na Tabela 9.

Observou-se que os valores de Alfa de Cronbach (A.C.) ou Confiabilidade Composta (C.C.) ficaram acima de 0,60, evidenciando assim a confiabilidade dos mesmos. $\mathrm{O}$ valor de $\mathrm{KMO}$ foi igual a 0,50, o que indica que o ajuste da Análise Fatorial foi adequado. De acordo com o critério Acceleration Factor, o constructo foi unidimensional.
Houve validação convergente do constructo, visto que a AVE foi superior a 0,40 .

\section{Descrição dos indicadores de tercei- ra ordem}

Ao verificar os constructos de terceira ordem, observa-se a seguinte análise fatorial, seus respectivos desvios padrões e seu intervalo de confiança:

Verifica-se que o indicador Percepções Gerais de Qualidade apresentou uma média de 6,02 com um desvio padrão de 0,68, o que pode se concluir que os consumidores concordaram, de uma forma geral, com o item apresentado.

\section{Modelo Teórico Hipotético}

Após as análises acima descritas, com as

TABELA 8 - Análise Fatorial do constructo de terceira ordem em pesquisa realizada com clientes bancários em 2018

\begin{tabular}{l|l|c|c|c}
\hline \multicolumn{2}{l|}{ Indicadores } & C.F. $^{1}$ & Com. $^{2}$ & Peso $^{2}$ \\
\hline \multirow{2}{*}{ Percepções Gerais de Qualidade } & Percepções Sistêmicas & 0,91 & 0,83 & 0,55 \\
\cline { 2 - 5 } & Percepções de Interação & 0,91 & 0,83 & 0,55 \\
\hline
\end{tabular}

${ }^{1}$ Carga Fatorial; ${ }^{2}$ Comunalidade.

Fonte: Elaborado pelos autores (2019).

TABELA 9 - Validação do constructo de terceira ordem em pesquisa realizada com clientes bancários em 2018

\begin{tabular}{|c|c|c|c|c|c|c|}
\hline Indicador de $3^{\mathrm{a}}$ ordem & Itens & A.C. ${ }^{1}$ & C.C. ${ }^{2}$ & $\mathrm{KMO}^{3}$ & $\operatorname{Dim}^{4}$ & AVE $^{5}$ \\
\hline Percepções Gerais de Qualidade & 2 & 0,80 & 0,85 & 0,50 & 1 & 0,83 \\
\hline
\end{tabular}

${ }^{1}$ Alfa de Cronbach; ${ }^{2}$ Confiabilidade Composta; ${ }^{3}$ Critério de Kaiser-Meyer-Olkin; 4Dimensionalidade; 5Variância Extraída; 6 Variância Compartilha Máxima.

Fonte: Elaborado pelos autores (2019).

TABELA 10 - Descrição do indicador de terceira ordem em pesquisa realizada com clientes bancários em 2018

\begin{tabular}{l|c|c|c}
\hline Indicador & Média & D.P. & I.C. - 95\% \\
\hline Percepções Gerais de Qualidade & 6,02 & 0,68 & {$[5,95 ; 6,09]$} \\
\hline
\end{tabular}

Fonte: Elaborado pelos autores (2019). 
especificações e visualizações de primeira, segunda e terceira ordem, torna-se possível a confirmação do modelo teórico, com suas respectivas cargas, conforme verifica-se abaixo:

Com este modelo teórico, pode-se entender que no campo das Percepções Sistêmicas, os itens Segurança e Confiabilidade apresentam estatisticamente o mesmo peso, tendo o item Eficiência uma menor relevância que os demais itens deste constructo. Os clientes se mostram mais preocupados com a eficácia dos sistemas de controles de segurança e a confiabilidade dos aplicativos e seus processos de interação, em comparação com a eficiência destes aplicativos no que se refere a questões técnicas, como espaço utilizado, tempo de resposta do app, consumo de energia, dentre outras situações.
Apesar de maior valoração, as cargas ainda se mostram próximas, ou seja, não obstante os clientes priorizarem a segurança e a confiabilidade dos sistemas de informação, a eficiência se mostra importante na percepção sistêmica dos consumidores. Assim se mostra viável às empresas bancárias direcionarem esforços para a evolução destes aplicativos, focando em sistemas cada vez mais ágeis e eficazes, mas sempre com um olhar especial para que esta evolução não abra brechas para a redução da segurança das operações.

Já no campo das Percepções de Interação, o item Facilidade apresentou um maior peso, sendo seguido pela Comunicação e com menor relevância o item de Comodidade. A facilidade na utilização dos aplicativos bancários se mostra essencial na interação

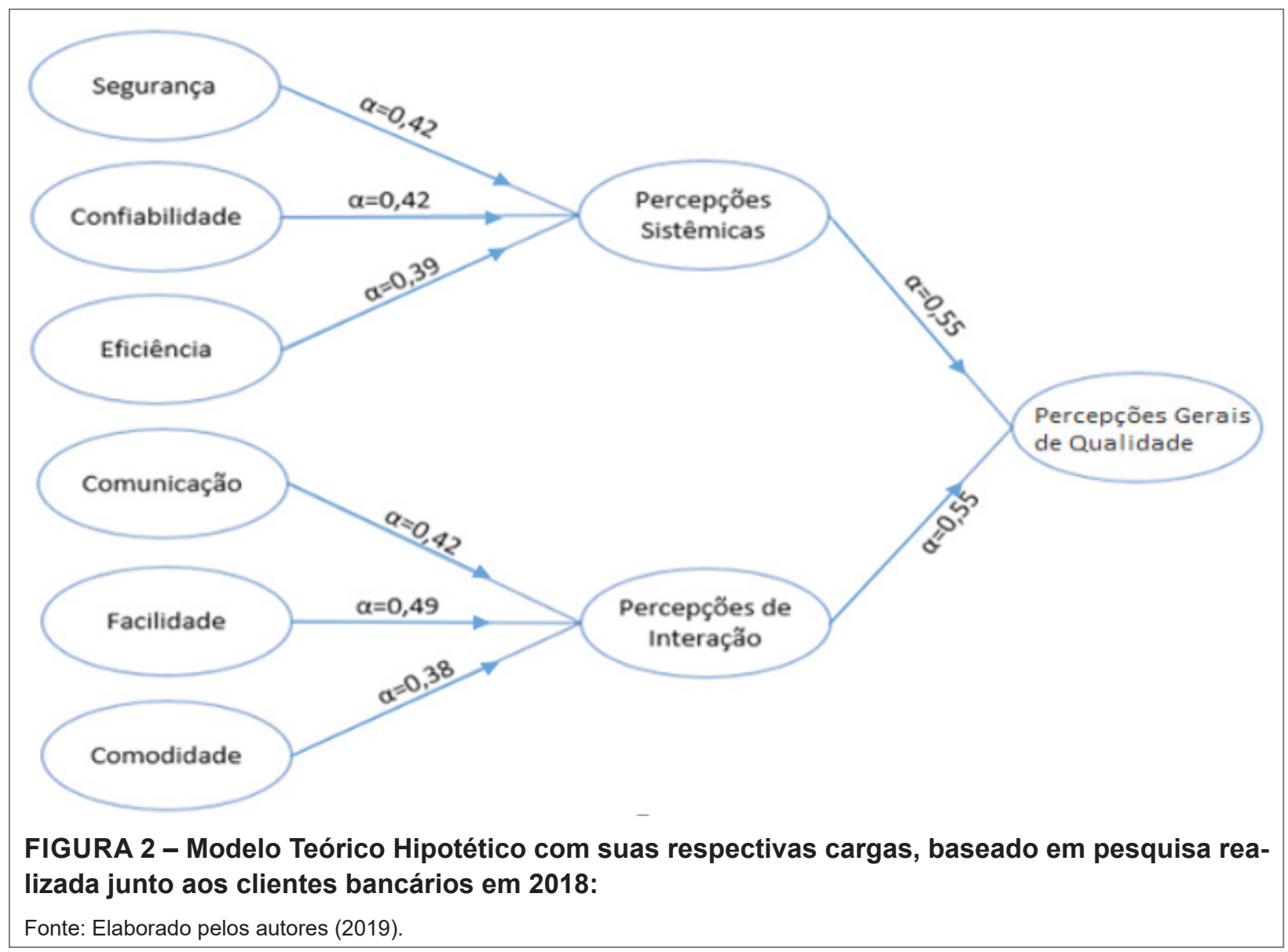

Fonte: Elaborado pelos autores (2019). 
com os clientes, sendo de grande importância no processo de decisão dos consumidores em utilizar os aplicativos bancários.

O processo de comunicação também se apresenta como importante na utilização dos app's, sentindo os clientes que a interação com as instituições financeiras através desta ferramenta é um valor percebido.A pesar dos clientes concordarem sobre a conveniência da comodidade, este se mostra menos importante pelos consumidores em comparação com os demais itens analisados.

Este estudo mostrou que os consumidores tendem a valorar a tranquilidade e facilidade em navegar pelos aplicativos bancários, sendo que se o aplicativo apresentar aspectos de usabilidade confusos ou complicados, os clientes tendem a abrir mão da sua comodidade. No que se refere ao processo de comunicação, este também se mostra com um peso maior, visto que caso o aplicativo apresente problemas de contato ou transmissão de dados, a comodidade novamente é suprimida. Finalizando, percebeu-se que os clientes tendem a abdicar de um conforto em realizar suas transações em qualquer lugar e em qualquer hora, caso os aplicativos não sejam simples, de fácil navegabilidade ou de comunicação ineficaz.

\section{CONSIDERAÇÕES FINAIS}

Percebeu-se nesta pesquisa que as tecnologias da informação e comunicação modificaram a forma de comunicar entre os bancos e seus clientes nos últimos anos e com o aumento constante de usuários de celulares e estes consumidores com acesso à internet, a tendência é a cada ano a quantidade de clientes bancários aderirem mais as tecnologias dos aplicativos. Com este crescimento, as instituições financeiras tendem a precisar inovar os processos existentes, acompanhando as novas tecnologias que surgirão, assim como as novas necessidades dos clientes, que a cada dia se tornam mais exigentes.

Tendo por base a pesquisa realizada e com este modelo teórico criado, verificouse a percepção dos clientes quanto aos aspectos de qualidade identificadas, entendendo as variáveis mais importantes e que os clientes apresentam maior valoração, bem como sua importância dentro do ciclo de inovação de uma instituição financeira, visto que, conhecendo em detalhe estas variáveis e suas potencialidades, pode-se criar novas perspectivas de crescimento.

Após todo o processo de análise, percebeu-se que, de uma forma geral, os consumidores de aplicativos bancários tendem a concordar sobre a importância da Segurança, Confiabilidade, Eficiência, Comunicação, Facilidade e Comunicação destes aplicativos, onde apresentam uma maior média de concordância nos itens de Comodidade e Segurança e uma menor média nos itens de Eficiência e Comunicação.

Ressalta-se que apesar desta maior valoração à segurança e confiabilidade, os consumidores entendem que a eficiência é um ponto importante, onde há a necessidade das empresas bancárias em investir em sistemas ágeis e simples, desde que esta evolução não crie uma vulnerabilidade destes aplicativos. Este resultado contribui junto ao entendimento trabalhado por Burt (1993), Carvalho, Serio e Vasconcellos (20I2), Granovetter (1973), Kaufman (2012) e Power (20l4), visto que se faz necessário uma constante busca de novas tecnologias e de integração com outros sistemas operacionais, visando uma melhor interação entre os consumidores e os app's.

Outro ponto importante neste proces- 
so de interação entre os consumidores e as instituições bancárias trata-se da maior valoração à facilidade do manuseio dos aplicativos, com peso superior à comunicação e à comodidade, o que demonstra que os clientes bancários ainda querem um sistema simples e de fácil usabilidade, sendo este quesito essencial para a decisão de utilizar estes aplicativos. Os aplicativos devem ser tanto inovadores quanto amigáveis.

Este ponto da pesquisa complementa a percepção de Kappel, Arruda e Pimenta (20 I4), Bombonatti Filho et. al., (20 I3), Heo et al.; (2009), Olsina, Santos e Lew (2014), Goel, Nagpal e Mehrotra (20 I8), Fiebig, Freitas (20I I), Siqueira Neto, Barcelos e Costa (20l8), na medida que a facilidade na utilização dos aplicativos se torna um diferencial para sua utilização pelos clientes.Assim, para a busca de migrar consumidores não aderentes a esta tecnologia, faz-se necessária a procura de tornar o sistema de interação cada vez mais simples e descomplicado.

Um ponto importante analisado no decorrer desta pesquisa trata-se da comodidade destas operações realizadas pelos app's, visto que apesar dos clientes entenderem como valor poder realizar suas demandas em seus celulares, independentemente do local que se encontrem, este item se mostra menos importante em comparação com o item facilidade, ou seja, caso o aplicativo não se mostre simples e descomplicado, os clientes podem tender a buscar os sistemas antigos de atendimento, como o próprio atendimento presencial. Assim, apesar de ser um item importante, caso o aplicativo não seja de fácil usabilidade, corre-se o risco dos clientes não aderirem a esta forma de atendimento remoto.

Por fim, esta pesquisa sinaliza que as instituições bancárias devem a cada dia se preocupar com a evolução dos seus aplicativos, visando torná-los mais simples para atender a todos os seus públicos, evoluindo seus sistemas de comunicação e sua eficiência, gerando uma maior comodidade para seus clientes, mas não se esquecendo de garantir sempre um processo de confiança com seus sistemas de segurança que garantam a confiabilidade das operações realizadas. Os aplicativos bancários são uma realidade na interação entre os bancos e seus consumidores, onde há a necessidade das instituições bancárias em investir cada vez mais em tecnologias para atender aos anseios diversos de seus clientes, visando, com isto, atender a uma demanda de mercado e, aproveitando desta onda de digitalização, em também reduzir seus custos e aumentar sua produtividade, criando possibilidades de apresentar um aumento de suas margens de lucros e ainda atender seus clientes de forma mais simples, ágil e eficaz. 


\section{REFERÊNCIAS}

ALBERTIN, Alberto Luiz; ALBERTIN, Rosa Maria de Moura. Dimensões do uso de tecnologia da informação: um instrumento de diagnóstico e análise. Revista de Administração Pública-RAP, v. 46, n. I, 2012.

BACEN. Disponível em: <https:// www3.bcb.gov.br/ranking/?wicket:interface $=:|:|>$. Acesso em: 5 fev. 2018.

BOMBONATTI FILHO, Oscar et al. Estratégia de Investimentos em Tecnologia da Informação e Comunicação e a Evolução da Indústria Bancária Brasileira:Análise Geral e dos Maiores Bancos Privados. Revista Gestão \& Tecnologia, v. I3, n. I, p. I5I176, 2013.

BURT, Ronald S. The social structure of competition. Explorations in economic sociology, v. 65, p. 103, 1993.

CARVALHO, Luciano Castro De; SERIO, Luiz Carlos Di; VASCONCELLOS, Marcos Augusto De. Competitividade das nações: análise da métrica utilizada pelo World Economic Forum. RAE-Revista de Administração de Empresas, v. 52, n. 4, 2012. Disponível em: <http://www.redalyc. org/html//55 I/I55 I 23 | 230 I I/>. Acesso em: I5 jul. 2017.

FEBRABAN. Pesquisa FEBRABAN de Tecnologia Bancária 2017. Disponível em: <https://cmsportal. febraban.org.br/Arquivos/documentos/PDF/Pesquisa\%20FEBRABAN\%20de\%20Tecnologia\%20 Banc\%C3\%Al ria\%2020 I 7.pdf>. Acesso em: 20 jul. 2017.

FEBRABAN. Pesquisa Febraban de Tecnologia Bancária 2018. Disponível em: <https://cmsportal. febraban.org.br/Arquivos/documentos/PDF/febraban_2018_Final.pdf>. Acesso em: 5 mar. 2019.
FIEBIG, Émerson Adriano; FREITAS, Ernani Cesar De. Canais de atendimento, satisfação e lucratividade de clientes em serviços: um caso bancário. REAd-Revista Eletrônica de Administração, v. I7, n. 3, 20II. Disponível em: <http://www.redalyc.org/ $\mathrm{html} / 40$ I I/40 I | 375 l 9007/>. Acesso em: 28 set. 2017.

FORNELL, Claes; LARCKER, David F. Evaluating Structural Equation Models with Unobservable Variables and Measurement Error. Journal of Marketing Research, v. I8, n. I, p. 39-50, I98I.

GERHARDT, Tatiana Engel; SILVEIRA, Denise Tolfo. Métodos de Pesquisa. Rio Grande do Sul: Plageder, 2009.

GIL, Antônio Carlos. Métodos e técnicas de pesquisa social. São Paulo:Atlas, 2008.

GOEL, Sakshi; NAGPAL, Renuka; MEHROTRA, Deepti. Mobile Applications Usability Parameters: Taking an Insight View. p. 3543, 2018.

GRANOVETTER, Mark S. The Strength of Weak Ties. American Journal of Sociology, v. 78, n. 6, p. I360-1380, maio 1973.

HEO, Jeongyun et al. A framework for evaluating the usability of mobile phones based on multi-level, hierarchical model of usability factors. Interacting with Computers, v. 21 , n. 4, p. 263-275, I ago. 2009.

KAPLAN, Andreas M.; HAENLEIN, Michael. Users of the world, unite! The challenges and opportunities of Social Media. Business Horizons, v. 53, n. I, p. 59-68, jan. 2010. KAPPEL, Lucas Borges; ARRUDA, Daniela Vasconcelos; PIMENTA, Márcio Lopes. Gestão de serviços bancários: Os motivos da não utilização do internet banking e do autoatendimento no interior do Brasil Central. REA-Revista Eletrônica de Administração, v. I3, n. I, p. 30-49, 20 I4.

KAUFMAN, Dora. A força dos "laços fracos” de Mark Granovetter no ambiente do ciberespaço. Galaxia, n. 23, 2012. Disponível em: <http://www.redalyc.org/ $\mathrm{html} / 3996 / 39964$ l 2490 | 7/>. Acesso em: 25 jun. 2017.

LOPES, Herton Castiglioni. O modelo estrutura-conduta-desempenho e a teoria evolucionária neoschumpeteriana: uma proposta de integração teórica. Revista de Economia Contemporânea, v. 20, n. 2, p. 336-358, ago. 2016.

MAINETTI JUNIOR, Sergio; GRAMANI, Maria Cristina Nogueira; BARROS, Henrique M. Despesas com tecnologia da informação e eficiência organizacional: novas evidências do setor bancário brasileiro. Review of Administration and Innovation - RAI, v. I I, n. I, p. I38, I 3 abr. 20 I4.

MALAQUIAS, Rodrigo Fernandes; ALBERTIN, Alberto Luiz. Por que os Gestores Postergam Investimentos em Tecnologia da Informação? Um Estudo de Caso/Why Do Managers Postpone Investments in Information Technology? A Case Study. Revista de Administração Contemporânea, v. I5, n. 6, p. I I20, 20 I I.

MARCONI, Marina de Andrade; LAKATOS, Eva Maria. Fundamentos de Metodologia Científica. Edição: 8 ed. São Paulo:Atlas, 2017.

OLSINA, Luis; SANTOS, Lucas; LEW, Philip. Evaluating Mobileapp Usability: A Holistic Quality Approach. Lecture Notes in Computer Science, 2014, Toulouse. Anais... Toulouse: Springer International Publishing, 2014. p. III-129. 
POWER, Dave. The Curve Ahead: Discovering the Path to Unlimited Growth. Edição: 2014 ed. New York: Palgrave MacMillan, 2014

SCHERER, Frederic M. Industry Structure, Strategy \& Public Policy. Nova York: HarperCollins College Publishers, 1996.

SCHUMPETER, J. A. Capitalismo

Socialismo e Democracia. St
Leonards: George Allen e Unwin Ltd, 1942.

SIQUEIRA NETO, Antônio Soares De; BARCELOS, M. T. C; COSTA, D. M. Perspectivas e percepções da inovação no mercado dos aplicativos bancários. Desafio Online, v. 6, n. I, p. $18,2018$.

STEVENSON, William J. Estatística aplicada à administração. São Paulo: Harbra, 200I.
TESTA, Mauricio Gregianin; LUCIANO, Edimara Mezzomo; JAEGER NETO, José Ignácio. Identificando - Potencial de Inovação das organizações por meio da análise do portfólio de projetos de Tecnologia da Informação. Gestão \& Produção (UFSCAR. Impresso), 2013.

YIN, Robert K. Estudo de Caso - Planejamento e Métodos. 5. ed. Porto Alegre: Bookman Editora, 2015. 